systeme. Insbesondere werden das räumliche Multiplexen zur Erhöhung der Übertragungskapazitäten ebenso wie der Einsatz für Paketdaten-Hochgeschwindigkeitsübertragungen in Mobilfunknetzen der 3. Generation, z. B. bei UMTS, und für Wireless LAN angeführt. Eine neue Methode zur Reduktion der Hardwarekomplexität von MIMO-Systemen wird vorgestellt. MIMO-Funkübertragungssysteme mit räumlichem Multiplexen werden auch in der Arbeit von Dominik Seethaler, Harald Artés und Franz Hlawatsch untersucht. Es wird nachgewiesen, dass mit Kugelprojektionsdetektoren bei ungünstigen Übertragungsbedingungen fast optimale Detektionsleistungen erzielbar sind.

Die Steigerung der Übertragungskapazitäten aus dem UMTS-Netz zum Teilnehmer ist für den erfolgreichen praktischen Einsatz von Hochgeschwindigkeitsdiensten, wie beispielsweise Web-Browsing oder Videostreaming, unabdingbar. Thomas Baumgartner kommt in seinem Aufsatz zu dem Schluss, dass zu dieser Kapazitätssteigerung in den UMTSNetzen mit Frequency Division Duplex (FDD) die so genannte Switched Beam-Methode wegen des geringeren Implementierungsaufwands dem Beam Pointing vorzuziehen ist, wenngleich beide Methoden einen $170 \%$ igen Kapazitätsgewinn gegenüber dem konventionellen System mit drei Sektoren erzielen.

Für die kostengünstige Bereitstellung neuer Dienste in den mobilen Netzen UMTS oder VoIP/IP-Multimedia-Subsystem IMS gehen Günther Pospischil, Igor Miladinovic und Harald Kunczier zunächst vom Intelligenten Netz (IN) aus, welches sich bei der Sprachtelefonie voll bewährt hat, und stellen Vergleiche zu SIP-basierten IN-Lösungen auf Basis der erweiterten Anforderungen an. Demgegenüber werden Lösungen mit Location Based Services diskutiert.

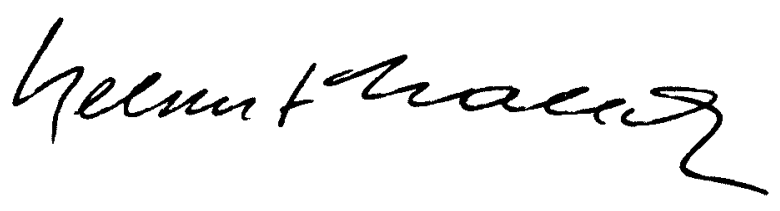

Helmut Malleck
Abschließend stellen Thomas Neubauer und Martin Toeltsch dar, dass sich die bestehenden Netzplanungstechniken nur bedingt von der 2 . auf die 3 . Mobilfunkgeneration übertragen lassen. So können die UMTS-Netzplanung und -optimierung - entsprechend ihrer überragenden wirtschaftlichen Bedeutung - nur mit gegenüber GSM deutlich erweiterten Methoden erfolgreich vorangetrieben werden.

Es verdient unsere uneingeschränkte Bewunderung, wie weit gestreut also die Angelpunkte des Erfolgs von Ernst Bonek sind. Sind es mathematische Modellierung und Simulation, physikalische Versuche und Tests sowie Projektierung auf der einen, so sind es human aspects zur Hochfrequenztechnik, sein Engagement für die Hochschulpolitik und in der Industrie auf der anderen Seite. Nicht zuletzt ist seine Unterstützung des OVE, zuletzt während entscheidender Phasen zur "networks 2004", dankend anzuerkennen!

Ernst Bonek als Persönlichkeit ist klar zu charakterisieren, denn es ist offenkundig, wie diszipliniert und ökonomisch der strenge Denker seine Kräfte einteilt. Und das führt uns zum zweiten, privaten Standbein von unserem Ernst, dem Orientierungslauf, bei dem er jede Etappe mit unterschiedlicher, optimal angepasster Kraftanstrengung meistert und somit viele Kollegen für seinen Sport begeistern kann. Alle, die Ernst Bonek zum Freund haben, wissen, dass er immer zur Stelle ist, um zu helfen - mit seiner weiten Sicht der Welt. Wir wollen inm mit dem vorliegenden Heft einen kleinen Teil der Freude zurückgeben, die er allen, die ihn kennen, täglich schenkt.

Allen Lesern recht viel Freude bei der Lektüre dieses Sonderhefts!

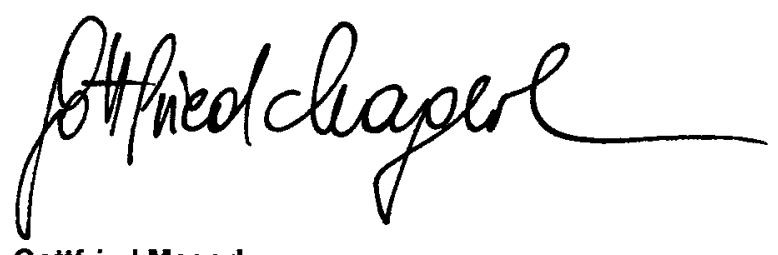

Gottfried Magerl

\title{
Was wäre die Mobilkommunikation ohne Ernst Bonek?
}

Zwischen dem österreichischen Handy-High-Level-Standard und Univ.-Prof. Dr. Ernst Bonek gibt es - so behaupte ich einen unleugbaren katusalen Zusammenhang.

Es war im Jahr 1986, als ich - damals Diplomingenieur der Elektrotechnik - aus meiner Heimatstadt Sarajewo nach Wien kam, um bei Ernst Bonek meine Dissertation zu schreiben. Ich war einer der ersten Assistenten jenes Professors, der an der Technischen Universität Wien für sein Engagement im Mobilfunk geschätzt war. Jede seiner Vorlesungen war ein Kommittment zum Mobilfunk, in einer Zeit, als das Festnetz noch das bestimmende Thema der Telekommunikation war. Damals steckte der Mobilfunk in seinen Kinderschuhen: Die damalige
C-Netz-Technologie, eine analoge, hatte gerade einmal einige 10000 Teilnehmer zu verzeichnen - Geschäftsleute, Vertreter öffentlicher Institutionen, die "ihr Handy" nur im Auto nutzen konnten.

In Wien gab es damals eine Handvoll Leute, die einfach mehr mit dem Mobilfunk erreichen wollten. Sie waren alle angesiedelt in der so genannten Generaldirektion der Post- und Telegraphen-Verwaltung (PTV), die damals Sektion des Bundesministeriums für Verkehr war. Diese Handvoll leitender Beamten wollte dem Mobilfunk zu einer Breitenwirkung verhelfen. Und da gab es noch Prof. Ernst Bonek: Er hatte hinsichtlich der Mobilkommunikation eine Vision. 
Seine Überzeugungskraft und seine nicht enden wollende Beharrlichkeit haben die PTV schließlich dazu bewogen, für die Finanzierung und fachliche Unterstützung eines Forschungsprojektes Mobilfunk an der TU Wien aufzukommen. Eine Premiere für die PTV (nie zuvor hat es eine so weit reichende Kooperation mit einer Forschungseinrichtung gegeben!) - eine Riesen-Chance für den österreichischen Mobilfunk - und eine tolle Herausforderung für mich: die dazugehörige Vertragsassistentenstelle wurde meine.

Als - mittlerweile - Generaldirektor des führenden österreichischen Mobilfunkbetreibers kann ich nicht aufhören zu betonen, dass Technik und deren Anwendungen keinen Selbstzweck darstellen, sondern von Menschen für Menschen gemacht sind. Eine Technologie (weiter) zu entwickeln, die Menschen ein mobileres Leben ermöglicht, das war die Aufgabe für mich. Dazu braucht es ein freundliches Umfeld mit freundschaftlichen Beziehungen - und das Gefühl „zu Hause" zu sein. Ernst Bonek hat mir diese Heimat gegeben. In den fünf Jahren meiner Forschungstätigkeit hat er mich nicht nur in meiner fachlichen Tätigkeit unterstützt, sondern mir geholfen, in diese österreichische Gesellschaft, der meine Großmutter, nicht aber meine Eltern und ich angehört haben, hineinzuwachsen.

Unvergesslich sind für mich nicht nur unsere fachlichen Auseinandersetzungen, in denen er sich dem oftmals „bockigen Kroaten" - der ich im Übrigen immer noch bin - als grandioser, weil auch problemlösender Sparringpartner zur Verfügung gestellt hat. Nicht vergessen kann ich, auch weil es meine Bilderwelt, meine Denke geprägt hat, die Geduld, mit der mir Ernst Bonek die Feinheiten der deutschen Sprache und der Präsentationstechnik näher gebracht hat. Ich, der ich bis dahin eher „Küchendeutsch" gesprochen habe, bin in dieser Forschungszeit für Mobilkommunikation um eine (Sprach)-Welt reicher geworden.

Wer Ernst Bonek auch nur einmal (!) gesehen hat, weiß, dass er kein steifer Wissenschaftler ist, der im Werken in schummrigen Labors seine einzige Bestimmung sieht. Ernst Bonek hat mich auch motiviert, neben der Doppelbelastung in der Industrie und am Institut aktiv Sport zu betreiben - ob bei spielerischem Training mit der Community seiner Sportsfreunde vom Orientierungslauf oder bei aktivem Handballtraining.

Auf einer Forschungsreise in den USA haben wir im Yosemite-Nationalpark selbstverständlich im Zelt übernachtet. Seine Grizzley-Bären-Prävention („Du darfst einfach keine Schokolade bei dir haben") ist mir ebenso unvergesslich wie viele andere Erlebnisse mit inm.

Anfang der 1990er-Jahre sind mit der Einführung des analogen D-Netzes auch die ersten tragbaren Handys auf den Markt gekommen - und damit ist die Attraktivität der Mobilkommunikation gewaltig gestiegen. Die Nutzeranzahl hat sich gegenüber dem C-Netz vervielfacht. Mittlerweile waren auch sehr viele Privatkunden im „Handy-Boot“.

Gleichzeitig wurde aber bereits an der nächsten Generation der Mobilfunktechnologie gearbeitet, der GSM-Technologie. An deren Standardisierung im Rahmen europäischer Forschungsprojekte (COST-Projekte) hat Ernst Bonek entscheidend mitgewirkt. Nicht nur, dass dieses Projekt - aus sicherer Distanz betrachtet einen Meilenstein der Telekommunikationsgeschichte darstellt, es war auch für mich ein Meilenstein in der wissenschaftlichen Zusammenarbeit mit meinem Mentor. Ewig in Erinnerung bleiben wird mir unser gemeinsames ZimmerErlebnis in Bern: Die Wirtin hatte meine Zimmer-Order verschlampt, Bonek hat meine Umquartierung verhindert, mir Platz in seinem Zimmer zur Verfügung gestellt - und mir die halbe Nacht über dabei geholfen, meine Präsentation vor der internationalen COST 231-Tagung am nächsten Tag zu perfektionieren. Sie sollte - weit über die Veranstaltung hinaus - internationalen Widerhall erreichen.

Aber was wäre die reine Forschung gewesen, ohne ihre Praktizierbarkeit! Nach meinem Gang in die Wirtschaft, zu Ascom und dann als Chef-Netzplaner zu A1, habe ich erkannt, wie sehr mir Ernst Bonek auch die praktische Seite der Forschung mitgegeben hat. Vieles wäre mir ohne die strenge und zugleich fürsorgliche „Bonek-Schule“ nicht umsetzbar gewesen. Gleichzeitig war es die „Bonek-Schule“, die mir das Rüstzeug gegeben hat, auch die tückischsten Fragen des Aufsichtsrates - und er war eines seiner Mitglieder - zu beantworten.

Heute ist der österreichische Mobilfunkmarkt nicht nur der meist umkämpfte europaweit. Aus der Kaderschmiede des Prof. Bonek und seiner Kollegen von der TU Wien haben zahireiche Absolventen dazu beigetragen, den österreichischen Mobilfunk auch international zu einem Spitzenplatz in Innovation und Qualität zu verhelfen. Darüber hinaus finden sich „Bonek-Abgänger“ in vielen Spitzenpositionen der internationalen Telekommunikationsbranche. - Wir alle haben Ernst Bonek viel zu verdanken.

Nur einen Wunsch hat er mir bislang noch nicht erfülit: Er hat mich nie an die schönsten Plätze der Welt, nämlich nach Kroatien begleitet. Aber das hat er mir für seine ruhigere Zeit versprochen, und diese, glauben wir, hat er jetzt.
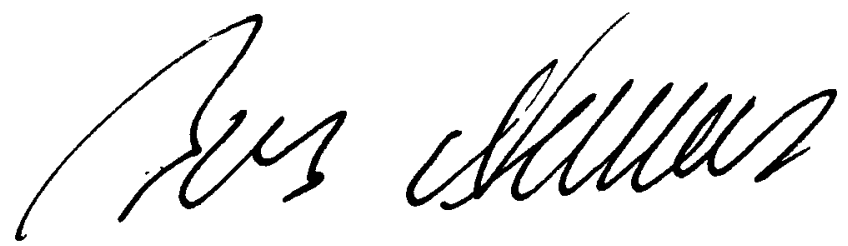

Boris Nemsic 